Progress Report: PermanganateTreatment of DNAPLs in Reactive Barriers and Source Zone Flooding Schemes

Contract Number: DE-FG07-96ER14735

Contractor:

Dr. Frank W. Schwartz

Department of Geological Sciences

The Ohio State University

$125 \mathrm{~S}$. Oval Mall

Columbus, $\mathrm{OH} 43210$

(614) 292-6196

\title{
Introduction
}

The goals of this study are (1) to elucidate the basic mechanisms by which potassium permanganate oxidizes common chlorinated solvents, various constituents in aqueous solution, and porous-medium solids, and (2) to assess the potential for chemical oxidation by potassium permanganate to serve as a remedial scheme involving either source zone flooding or reactive barriers. The research plan involves a combined experimental/modeling study that builds on our extensive previous work in the area of reactive barrier systems, and modeling of reactive contaminant transport. The experimental studies are being undertaken at The Ohio State University by Dr. Schwartz and his co-workers. The modeling work is being conducted in Albuquerque, NM by Dr. Zhang of Intera, Inc.

The workplan for this study is designed around the fol lowing four objectives (1) to describe through batch experiments the kinetics and mechanisms by which potassium permanganate oxidizes dissolved tetrachloroethene (PCE), trichloroethene (TCE), and dichl oroethene (DCE), (2) to exami ne using column studies the nature and kinetics of reactions between potassium permanganate, residual DNAPLs (PCE, TCE, and DCE) and porous medium solids, (3) to represent the process understanding in flow and transport models that demonstrate the potential applicability of the approach, and (4) to apply the resulting computer code in the devel opment of appropriate field tests for assessing the approach.

\section{Overview of Activities}

To date work there has been significant progress in addressing objectives 1 and 3. Batch studies have been completed to examine the rates of oxidation of PCE, TCE, DCE and several alcohols. A complimentary series of experiments have been completed to provide a preliminary evaluation of the reaction pathways by which chlorinated aliphatic compounds are oxidized. A chapter summarizing the results of these studies (Yan and Schwartz, 1997) has been recently submitted to J ournal of 
Contaminant Hydrology and an oral chapter is scheduled for presentation at the Geological Society of America Meeting in October (seelist of papers). A second chapter is now in preparation that looks at reaction pathways (i.e., intermediate products) in more detail and the significance of surface catalyzed oxidation. We expect that this chapter will be ready for submission in about two months.

Dr. Zhang has made excellent progress in the devel opment of the flow and transport code for thetransport of various chlorinated ethylenes, and their breakdown products. In its present form, the code accounts for multidimensional flow and multi-component transport of mobileand immobile constituents. Reactions involving TCE, $\mathrm{MnO}_{4}$; intermediates that form (e.g., formic acid, oxylic acid) and $\mathrm{CO}$, are represented kinetically. The code al so simulates the dissolution of DNAPL, as a function of saturation, to provide a source of dissolved cotaminants. The iterative scheme for solving the coupled flow and transport problem; and illustrative calculations involving a one-dimensional column system form the basis of an oral paper to be given at the Fall AGU Meeting. We expect that the first journal article stemming from this aspect of the study to come out in early next year.

\section{Significant Research Results}

This section outlines the most important research findings to date in the study. In general, chlorinated ethylenes can be rapidly degraded by permanganate in aqueous solution. The half-lives of TCE, cis-1,2-DCE , trans-1,2-DCE, and 1,1DCE reacting with $1 \mathrm{mM} \mathrm{MoO}$; is in a range from 0.4 to 18 minutes (Figure 1 ). The half-life for PCE degradation, however, is about four hours. In PCE, the attack of permanganate ion, as an electrophile, is sl owed by the deficiency of electrons in the carbon bond, induced by four chlorines in PCE.

Kinetic studies of TCE oxidation by permanganate indicate that TCE degradation is second order. The reaction can reasonably described as:

$$
\mathrm{C}_{2} \mathrm{HCl}_{3}+\mathrm{MnO}_{4}^{-} \stackrel{\xi_{1}}{\rightarrow} \mathrm{I} \stackrel{\xi_{2}}{\rightarrow} \mathrm{CA}+\mathrm{MnO}_{2}+3 \mathrm{Cl}^{-}
$$

where I is a cyclic complex (e.g., cyclic hypomanganate ester), CA are various carboxylic acids (e.g., glyoxylic acid, formic acid), $\mathbf{k}_{1}$ is a second-order rate constant, and $\mathbf{k}_{2}$ is an unknown-order rate constant. The rate constant $\mathbf{k}_{\mathbf{1}}$ is independent of $\mathrm{pH}$ over the range 4-8. However, the decomposition of I in the second step likely involves hydrogen ions. The reaction in the second step removes chlorine atoms 
relatively rapidly from the complex (I). Mass balance determinations through an experiment show that essentially all of the chlorine ends up as chloride ion in solution.

Spectrophotometric analysis of samples collected during a batch experiment were analyzed at wavel engths ranging from 400 to $700 \mathrm{~nm}$. Results indicate that the main reaction product is colloidal manganese dioxide $\left(\mathrm{MnO}_{2}\right)$. Neither $\mathrm{MnO}_{4}{ }^{2}$ nor $\mathrm{MnO}_{4}^{3 \cdot}$ appear to form as products. There is some indication that the $\mathrm{MnO}_{2}$ autocatalyzes reactions between TCE and $\mathrm{MnO}_{4}$ : We are now studying this effect in greater detail.

Permanganate is a powerful and reactive oxidant which is capable of reacting with other organic compounds in ground-water. As a practical matter at sites Involving contaminant mixtures, it is often difficult to characterize the contaminants fully. As an initial step to examine the influence of competition in the reactions, we undertook experiments involving ground water and contaminated ground water. Other organic compounds present in solution utilized $\mathrm{MnO}_{4}$ and reduced the destruction rate of the target organic contaminant (i.e., TCE) (Figure 2). It appears that the reaction kinetics of this more complicated contaminant system can be described using TOC as an indicator parameter.

To date, the main emphasis in modeling has been to develop the capabilities of the multi-component flow and transport code. Calculations are provided here to illustrate the capabilities of the code. The simple problem involves transport of a slug of aqueous TCE as it is displaced by $\mathrm{MnO}_{4}$. The onedimensional column is assumed to be $161.75 \mathrm{~m}$ long with a cross-sectional area of Im? The system of reactions describing the permanganate-TCE interaction is assumed to have the following form

$$
\left[\begin{array}{l}
k_{f 2} \\
k_{f 2} \\
k_{f 3} \\
k_{f 4}
\end{array}\right], \quad 0-\left[\begin{array}{ccccc}
-1 & -1 & 1 & 0 & 0 \\
-1 & -1 & 0 & 1 & 0 \\
0 & -1 & -1 & 0 & 1 \\
0 & -1 & 0 & -1 & 1
\end{array}\right]\left[\begin{array}{l}
A \\
B \\
C_{1} \\
C_{2} \\
C_{3}
\end{array}\right]
$$

where $k_{f j}(j=1, ., 4)$ is the forward kinetic rate constant, $A, B, C_{1}, C_{2}$ and $C_{3}$ represent chemical species TCE, $\mathrm{MnO}_{4} ; \mathrm{HCOOH},(\mathrm{COOH})_{2}$, and $\mathrm{CO}$, respectively.

Figure 3 shows partial results for a case described briefly on the figure. Both TCE and $\mathrm{MnO}_{4}$ concentrations decrease as a function of transport al ong the column. 


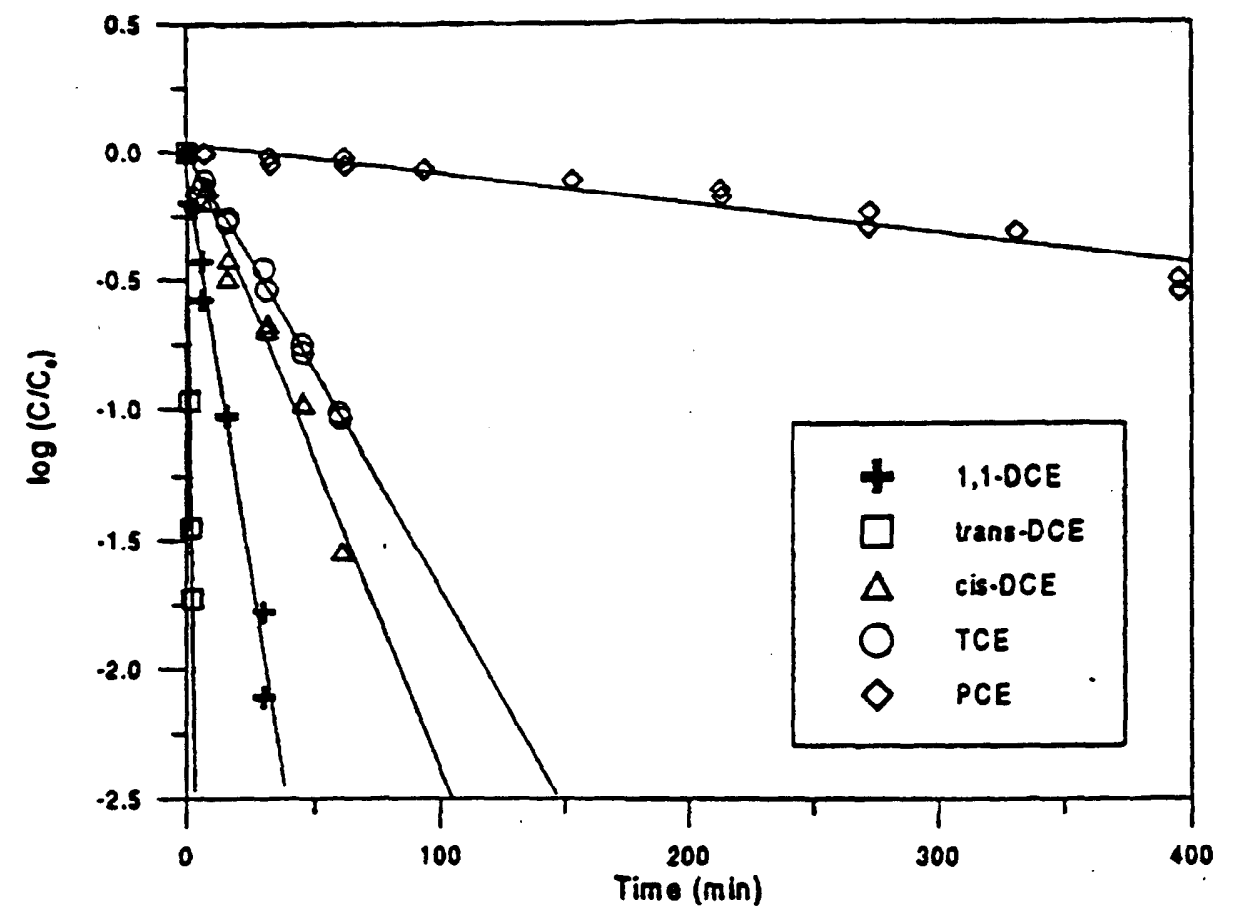

Figure 1. Degradation of chlorinated ethylenes by $\mathrm{MnO}_{\mathbf{a}^{-}}(1 \mathrm{mM})$ at $\mathrm{pH}$ 7.1. Lines represent best fits using a pseudo-first-order kinetic model.

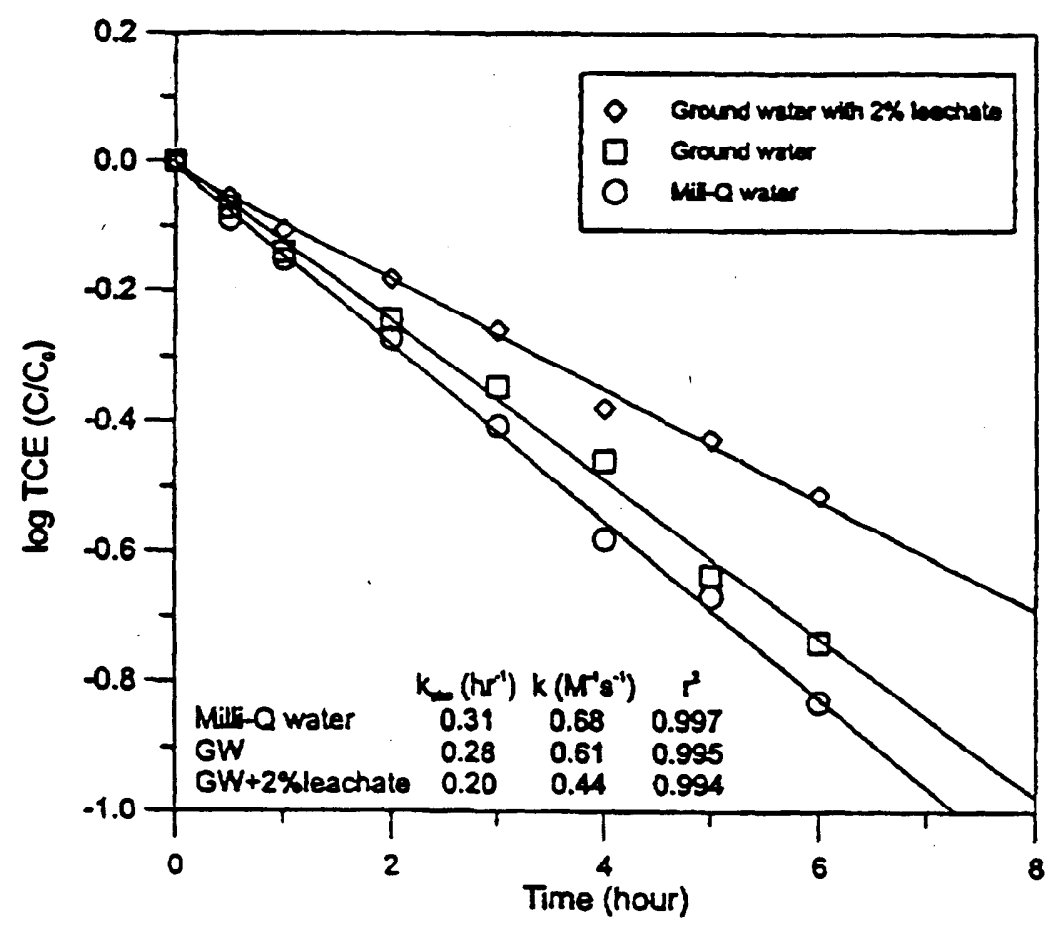

Figure $\overline{2}$. Comparison of TCE degradation by $0.13 \mathrm{mM}(20 \mathrm{mg} / \mathrm{L}) \mathrm{MnO}_{4}$ in Milli-Q water, ground water, and contaminated ground water ( $2 \%$ leachate). Lines represent best fits using a pseudo-first-order, kinetic equation. 


\section{Partial Summary of Run Parameters}

$h_{\text {ram }}=5.0 \mathrm{~m}, h_{2=161.75 \mathrm{~m}}=0.0 \mathrm{~m}$

$k_{\mathrm{a}}=0.001 \mathrm{~L} /$ moles $/ \mathrm{s}(\mathrm{i}=1, . ., 4)$

$C_{\text {MnO4 }}=1500 \mathrm{mg} / \mathrm{L} @ \mathrm{x}=0 \mathrm{~m} ; C_{T C E}=1000 \mathrm{mg} / \mathrm{L}$ for $x=(0.0,0.75 \mathrm{~m}) @ \mathrm{t}=0$

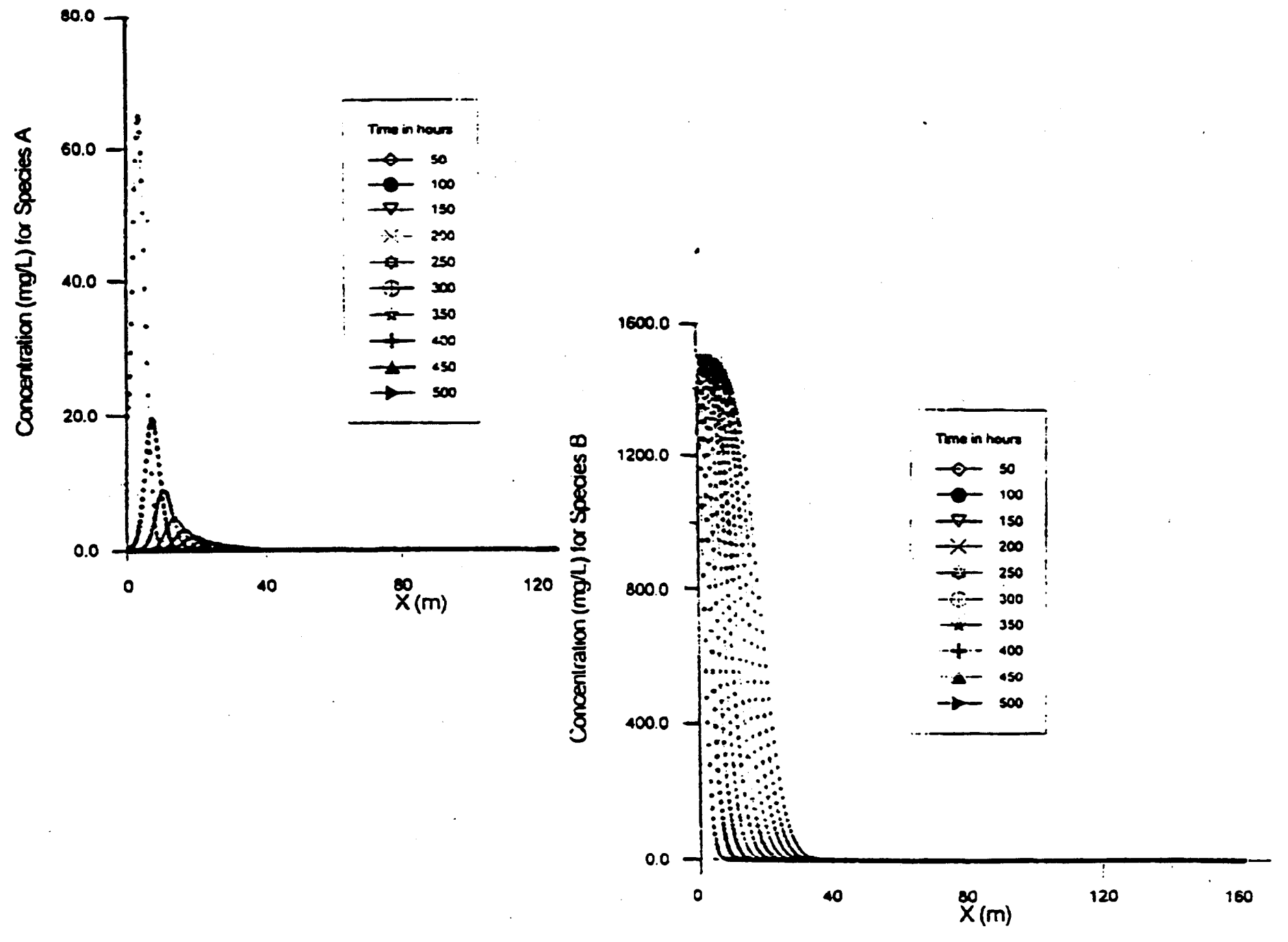

Figure 3. Illustrative model calculation for multi-component transport within a 1$\mathrm{D}$ column. Concentrations are depicted for Species A (TCE) and Species B $\left(\mathrm{MnO}_{4}\right)$. 


\section{Oral Presentations and J ournal Articles}

Yan, Y. E., and F.W. Schwartz, 1997. Oxidative degradation of chlorinated ethylenes by potassium permanganate: submitted toJ ournal of Contaminant Hydrology, (September 1997), 41 ms. pages.

Yan, Y.E., and F.W. Schwartz, 1997. In-situ oxidative degradation of chlorinated ethenes: oral presentation, Geol ogical Society of America, Annual Meeting Salt LakeCity.

Zhang, H., F.W. Schwartz, and Y.E. Yan, 1997. Simulation of permanganate treatment of DNAPLs in a source zone flooding scheme. American Geophysical Union, Fall Meeting, San Francisco. 